\title{
Reconstruction of Sound Source Pressures in an Enclosure Using the Phased Beam Tracing Method
}

Jeong, Cheol-Ho; Ih, Jeong-Guon

Published in:

Acoustical Society of America. Journal

Link to article, DOI:

$10.1121 / 1.3132502$

Publication date:

2009

Document Version

Publisher's PDF, also known as Version of record

Link back to DTU Orbit

Citation (APA):

Jeong, C-H., \& Ih, J-G. (2009). Reconstruction of Sound Source Pressures in an Enclosure Using the Phased Beam Tracing Method. Acoustical Society of America. Journal, 126(1), 158-166.

https://doi.org/10.1121/1.3132502

\section{General rights}

Copyright and moral rights for the publications made accessible in the public portal are retained by the authors and/or other copyright owners and it is a condition of accessing publications that users recognise and abide by the legal requirements associated with these rights.

- Users may download and print one copy of any publication from the public portal for the purpose of private study or research.

- You may not further distribute the material or use it for any profit-making activity or commercial gain

- You may freely distribute the URL identifying the publication in the public portal

If you believe that this document breaches copyright please contact us providing details, and we will remove access to the work immediately and investigate your claim. 


\title{
Reconstruction of sound source pressures in an enclosure using the phased beam tracing method
}

\author{
Cheol-Ho Jeong \\ Department of Electrical Engineering, Acoustic Technology, Technical University of Denmark, \\ DK-2800 Kongens Lyngby, Denmark \\ Jeong-Guon $\mathrm{Ih}^{\mathrm{a})}$ \\ Department of Mechanical Engineering, Center for Noise and Vibration Control (NoViC), KAIST, \\ Daejeon 305-701, Korea
}

(Received 15 September 2008; revised 15 April 2009; accepted 15 April 2009)

\begin{abstract}
Source identification in an enclosure is not an easy task due to complicated wave interference and wall reflections, in particular, at mid-high frequencies. In this study, a phased beam tracing method was applied to the reconstruction of source pressures inside an enclosure at medium frequencies. First, surfaces of an extended source are divided into reasonably small segments. From each source segment, one beam is projected into the field and all emitted beams are traced. Radiated beams from the source reach array sensors after traveling various paths including the wall reflections. Collecting all the pressure histories at the field points, source-observer relations can be constructed in a matrix-vector form for each frequency. By multiplying the measured field data with the pseudo-inverse of the calculated transfer function, one obtains the distribution of source pressure. An omni-directional sphere and a cubic source in a rectangular enclosure were taken as examples in the simulation tests. A reconstruction error was investigated by Monte Carlo simulation in terms of field point locations. When the source information was reconstructed by the present method, it was shown that the sound power of the source in an enclosure could be estimated. (C) 2009 Acoustical Society of America. [DOI: 10.1121/1.3132502]
\end{abstract}

PACS number(s): 43.40.Sk, 43.60.Jn, 43.55.Ka [NX]

Pages: $158-166$

\section{INTRODUCTION}

Acquiring information of acoustic parameters on a source surface such as normal velocity, acoustic pressure, impedance, intensity, and radiated sound power is very important in many acoustic problems. For example, for effective noise control, the identification of dominant vibroacoustic sources takes the top priority in the early stage.

Many techniques have been developed for the accurate identification and ranking of the acoustic sources. The coherence method uses the statistical signal processing technique, in which the relation between the measured data at source and receiver positions is analyzed to identify the linear dependency between them. ${ }^{1}$ This method is effective when the accurate numerical modeling of acoustic sources and transmission paths is difficult due to the complexity in boundary geometries and properties. In the sound intensity method, an acoustic energy flow in the sound field, usually at the near field of a radiator, is measured to identify the source and sink distribution around the source surface. ${ }^{2}$ However, the measured intensity is indeed a field property, not a source property. Therefore, strong normal active intensity data near a radiating surface do not actually and necessarily mean that the nearest radiator surface portion corresponds to a strong contributor. Practicing this method requires substantial time and effort to scan the sound field, in particular, in the mea-

\footnotetext{
a) Author to whom correspondence should be addressed. Electronic mail: j.g.Ih@kaist.ac.kr
}

surement for a large radiator or a fine spatial resolution. If tri-directional intensity data are measured at field points with a proper spatial resolution, reconstruction and visualization of the source might be possible. Owing to the recent progress, the near-field acoustic holography (NAH) becomes the most popular techniques in the source identification. Spatial Fourier transform of the spatial field data measured in the near-field to the wave number domain has been generally taken as a method to describe the sound propagation from a radiator to a regular-shaped hologram plane. ${ }^{3}$ By virtue of the rapid development of the computational capability, the boundary element method (BEM) to find out the vibroacoustic transfer function between the source surface and the field point has been widely used in the NAH. ${ }^{4,5}$ The beam forming is also an array-based technique for the sound source localization at the far field. ${ }^{6}$ This method does not require an array to be larger than the sound source. Furthermore, it can use an irregular array whereas the NAH usually requires a regular and rectangular sensor grid in the measurement. However, detection of coherent sources in the similar bearing angle is problematic and the precise estimation of the source parameters is somewhat tricky. A discrete Huygens model in rooms was suggested for source identification based on the time reversal process. ${ }^{7}$ Pressures at receiver locations go back to a source point where sound is emanated, by multiplying inversed transmission line matrices.

For a reconstruction of the source information at medium frequencies, the phased beam tracing method $(\mathrm{PBTM})^{8,9}$ is adequate to calculate acoustic transfer func- 
tions between source segments and receiver points. Once transfer functions are calculated by the PBTM, it is conceptually possible to recover a pressure distribution over the source surfaces by multiplying the measured field pressure data with the inverse transfer function. Similar problems with the NAH technique would arise in the matrix inversion process and the positioning of sensors. Numerical techniques for improving the singularity of the system matrix can be adopted to enhance the resolution of the reconstructed field.

This study aims at reconstructing the acoustic property of source surfaces in a room without moving it to an anechoic chamber. Theoretically, various holographic methods can be used for the reconstruction of the acoustic parameters on the source surface, but the modeling of all boundary surfaces of the room with precision is very difficult, in particular, at medium to high frequencies for large rooms. In this regard, it is thought that for a noise source in an enclosed room the PBTM can be a good alternative to inversely calculate surface pressures on sound sources. This paper mainly addresses the fundamental formulation, the numerical stability of applications, and the effects of field point locations.

\section{PHASE GEOMETRICAL ACOUSTICS}

First of all, the phased geometrical acoustics method is a modified geometrical acoustic technique for simulating sound propagation at medium to high frequency range by the aid of phase information. If the frequency of interest is far beyond the Schroeder cutoff frequency $f_{c},{ }^{10}$ the phase information is not really necessary because of heavy modal overlaps, which implies that each modal character cannot be clearly distinguished. In this range, the statistical models or geometrical acoustics have been successfully adopted. Among many geometrical acoustics techniques, image source methods can account for arbitrary geometry robustly, but it is limited to specular reflection. ${ }^{11,12}$ Conventional raytracing methods model specular or diffuse reflections and arbitrary room geometry efficiently, but a volume receiver should be employed. ${ }^{13,14}$ Due to the use of a volume receiver, responses of ray tracing are smeared temporally and spatially. Also discrete sampling of rays leads to undersampling errors, so enormous rays are needed to avoid sampling errors. ${ }^{15}$ Beam tracing methods can analyze room acoustics in a similar way as the ray tracing with spatially extended beams and a point receiver, but problems occurs when beams intersects more than one surface. ${ }^{8,9,16-19}$ If an intersecting polygon is detected, there are two solutions: The original beam is followed by its central axis ray ${ }^{8,9,16}$ or the original beam can be split. ${ }^{17,18}$ Splitting algorithms are robust and safe, but they become computationally voracious. However, conventional geometrical acoustics methods, which normally ignore phase and consider only energy quantities, cannot be used in the source identification at all.

At low frequencies, wave-based methods are the most reliable and appropriate tools in calculating transfer functions; thus the NAH based on wave-based methods is the core technique for reconstructing source information accurately. However, at around the Schroeder cutoff frequency $f_{c}$, both wave-based methods and high frequency methods can- not tackle acoustic problems appropriately: Wave-based methods require a lot of computational expenditure due to huge number of elements, whereas lack of phase information and modal characteristics leads high frequency methods to inaccurate outcomes. Therefore phased geometrical acoustics methods have been suggested. Inclusion of phase is twofold: phase at reflections from surfaces and propagation phase. Consequently outcomes of phased methods are sound pressures at receiver locations in time or frequency domain. Consequently the methods have been mainly used for calculating an impulse response or an acoustic transfer function for a source-receiver pair in simple rooms at medium frequencies. Initially phase was introduced into a ray-tracing model so that it could be applied to lower frequencies. ${ }^{20}$ Suh and Nelson $^{21}$ analyzed several rectangular rooms and obtained satisfactory results at early reflections using the phased image source method. Jeong et al. ${ }^{9}$ applied the phased beam tracing to predict impulse responses and acoustic parameters in a room. An improvement was found at medium frequencies in comparison with the conventional methods.

Wareing and Hodgson ${ }^{8}$ developed a transfer-matrix model integrated into a beam tracing method for multilayered surfaces. An adaptive beam tracing method was tested in Bell laboratories by Tsingos et al., ${ }^{22}$ yielding a remarkable agreement with measurements. In their simulation, they incorporated the uniform theory of diffraction ${ }^{23}$ with the beam tracing for invisible source-receiver pairs.

In this study, the triangular beam tracing approach proposed by Lewers ${ }^{16}$ was extended to include phase. The beam tracing algorithm consists of source generation, surfacegeometry definition, traces of beams, and receiver detection. Source division is based on an icosahedron, which makes the beam cross section an equilateral triangle. One edge of an equilateral triangle can be divided into $p$ equal lengths, resulting in a polygon with $20 p^{2}$ faces. Room surfaces should be planar, which are mathematically modeled as $A_{i} x+B_{i} y$ $+C_{i} z+D_{i}=0$. A trajectory of a beam is scanned by combined processes of determining the nearest plane, finding the new image source, calculating the reflected vector. Consider a beam, which is defined by a central axis and three boundary planes, each plane forming a side of the beam. Beams do not fragment on reflection and the direction after reflection is determined entirely by its central axis. Once the trajectory of the beam is identified, the possibility that a point receiver is surrounded by the beam boundary planes is tested using the normal vectors of the boundary walls. Following a positive receiver point test, the complex pressure amplitude for the beam is calculated and finally the transfer function is constructed.

Most published works have been tested in quite simple rooms, because of the inherent limitation of the geometrical acoustics methods. The most challenging task is to deal with wave phenomena, especially diffraction and diffuse reflection. Therefore, a room shape should be simple and wall surfaces should be relatively smooth and large. Highly faceted surfaces should be avoided and coarse models are adequate for the present method. If roughness of surfaces or dimensions of obstacles in rooms is much smaller than the 
wavelength of interest, these can be neglected in the modeling procedure. Scattering of smooth surfaces of the test room is ignored.

As mentioned earlier, diffraction is the most challenging topic in geometrical acoustics and it has been argued that phased geometrical acoustics methods without proper diffraction algorithms cannot correctly predict the room acoustics of enclosed rooms. According to Pierce, ${ }^{24}$ "amplitudes of the diffracted field are usually much weaker than direct and even reflected contributions." Diffraction was only considered in shadow regions in several previous works, assuming its contribution is relatively small in illuminated regions where direct and reflected contributions from a source also reach a listener. ${ }^{22,25}$ On the other hand, Torres et al. ${ }^{26}$ claimed that diffraction can be perceived in illuminated regions. It is still debatable if the diffraction should be included for entire enclosed sound field. According to the authors' experience, the present beam tracing algorithm neglecting diffraction can satisfactorily predict acoustic parameters and transfer functions in rooms with simple geometry. ${ }^{9,27}$ It is true that if receivers in rooms are distant from diffracting obstacles, edges, and corners, geometrical acoustics components are dominant compared to diffracted components. Actually finding diffraction paths is a very elaborate task and, moreover, diffraction of finite, non-rigid wedges still needs to be studied further. In this regard, diffraction has not been taken into account for observation points far from diffracting objects in simple rooms. However, the authors believe that incorporation of recently developed diffraction algorithms ${ }^{26,28,29}$ into phased geometrical acoustics models will perform better and more advanced phased geometrical acoustics methods will be successfully applied to source identification and reconstruction in the future.

\section{INVERSE ALGORITHM FOR THE PHASED BEAM TRACING METHOD}

The most important step in the source identification is the effective characterization of multiple transfer functions between a noise source and field points. A source surface radiating sound is modeled by appropriate boundary conditions, i.e., Neumann, Dirichlet, and mixed-type boundaries. Then, exactly speaking, the transfer function between source and field can be called a vibro-acoustic or a purely acoustic transfer function depending on the boundary condition type; however, a vibro-acoustic source is used in this paper assuming that the Neumann-type boundary, e.g., a hole, with a medium fluctuation can be also modeled as an equivalent vibrating source element like a moving piston as far as the size is small. Consider an extended vibro-acoustic source radiating sound waves into a large enclosure filled with homogeneous air medium. For modeling purposes, let the source surface, either actual or simplified one, be divided into small segments, called source segments. The resultant sound field in an enclosure is observed at many field points (or observation points). In order to obtain precisely restored results, over-determined field data are usually used for a uniformly distributed sensor points; that is, the number of field points $N$ is larger than the number of source segments $M$. However, this over-determined condition is not the necessary

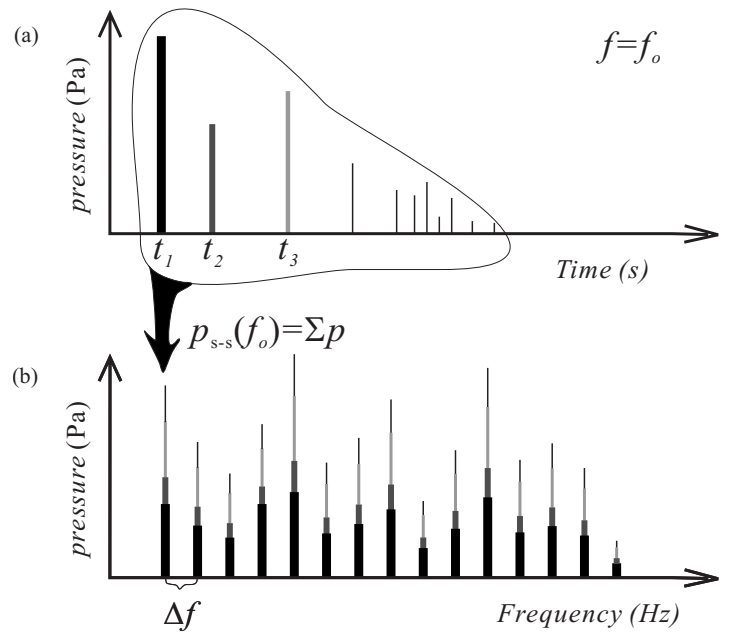

FIG. 1. Typical results by the phased beam tracing simulation. (a) Pressure reflectogram and (b) steady-state transfer function.

condition for the inverse reconstruction. If one can somehow select a very independent set of field points, usually unevenly distributed sensor positions, even an underdetermined condition of source and field data may be acceptable with a reasonable precision.

Using the PBTM, acoustic transfer functions between source segments and field points can be obtained. First, a pressure at a receiver by a beam is computed based on the information of the path length and surfaces that the beam hits, as

$$
p\left(t=\frac{a_{\mathrm{tot}}}{c_{o}} ; \theta_{i}, \omega\right)=\frac{p_{s, m}}{a_{\mathrm{tot}}} e^{-j \tilde{k} a_{\mathrm{tot}}} \prod_{i=1}^{q} r_{i}\left(\theta_{i}\right)
$$

where $p_{s, m}$ is the pressure amplitude at the $m$ th source segment, $a_{\mathrm{tot}}$ is the total travel distance of the beam, $\tilde{k}$ is the complex wave number (or $\tilde{k}=k-j 0.5 \mathrm{AF}$ ), $k$ is the wave number in a lossless free-field, AF is the attenuation factor of the air, $r_{i}\left(\theta_{i}\right)$ is the pressure reflection coefficient of the $i$ th wall reflection, $\theta_{i}$ is the angle of incidence of the beam to the $i$ th wall, $q$ is the total number of wall reflections until the beam reaches the receiver, and $c_{o}$ is the speed of sound in air. It is noted that the pressure in Eq. (1) is the complex pressure at the receiver point for a single frequency, and for the single beam departing from the $m$ th source segment. Figure 1(a) shows a pressure reflectogram (or an echogram) for a single frequency. For simplicity, absolute magnitude plots are shown in Fig. 1. The total steady-state acoustic pressure is calculated by summing the total pressures of all beams detected at the receiver point. In Fig. 1(b), a steady-state transfer function for a unit input (at the source) is shown by collecting all frequency components. At this point in calculation procedure, frequency range and frequency resolution should be determined. The bottom parts of the pressure magnitude bars, which are the thickest, denote the contributions by the direct sound. The next bottom parts show the contributions from the reflected pressures at time $t_{2}$, and the further next parts show the contributions from the reflected pressures at time $t_{3}$, and so on. 


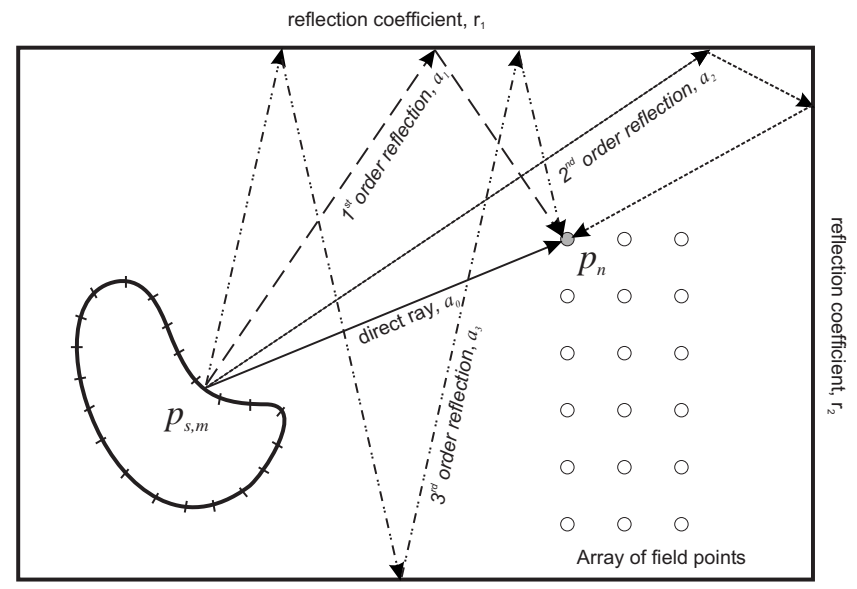

reflection coefficient, $r_{3}$

FIG. 2. A schematic two-dimensional presentation of the sound propagation within an enclosure. In the left, an extended source radiates the sound, of which the surface is comprised of $M$ segmental areas. The surface pressure at the $m$ th segment is expressed as $p_{s, m}$. Then, an array of $N$ sensors receives the direct and the reflected sounds. Here, $a_{i}$ denotes the travel distance of the $i$ th reflection and $r_{j}$ represents the reflection coefficient at the $j$ th wall.

Figure 2 illustrates a conceptual presentation of the sound radiation and propagation within a two-dimensional enclosure. An extended source, which is comprised of $M$ segmental surface areas, radiates sound. The surface pressure at the $m$ th segment is expressed as $p_{s, m}$. Then, an array of $N$ sensors receives the direct and the reflected sounds and the waves are superposed in time. In this figure, $a_{i}$ denotes the travel distance of the $i$ th reflection and $r_{j}$ represents the reflection coefficient at the $j$ th wall. Therefore, in notation, the zeroth reflection means the direct sound. In the actual situation, of course, three-dimensional geometrical modeling and scanning should be carried out to simulate the sound propagation in an enclosure. The field pressure at the $n$th field point caused by the $m$ th source segment, $p_{n}$, can be expressed as a sum of direct component and successive reflected components (up to $q$ th order) as follows:

$$
\begin{aligned}
p_{n}(\omega)= & p_{s, m} \frac{e^{-j(k+j 0.5 \mathrm{AF}) a_{o}}}{a_{o}}+p_{s, m} \frac{e^{-j(k+j 0.5 \mathrm{AF}) a_{1}}}{a_{1}} r_{1}(\theta)+\cdots \\
& +p_{s, m} \frac{e^{-j(k+j 0.5 \mathrm{AF}) a_{q}}}{a_{q}} \prod_{i=1}^{q} r_{i}(\theta) \\
= & {\left[\frac{e^{-j(k+j 0.5 \mathrm{AF}) a_{o}}}{a_{o}}+\frac{e^{-j(k+j 0.5 \mathrm{AF}) a_{1}}}{a_{1}} r_{1}(\theta)+\cdots\right.} \\
& \left.+\frac{e^{-j(k+j 0.5 \mathrm{AF}) a_{q}}}{a_{q}} \prod_{i=1}^{q} r_{i}(\theta)\right] p_{s, m}=H_{n m} p_{s, m} .
\end{aligned}
$$

When a sufficient number of reflections is counted, the steady-state transfer function, $H_{n m}$, between the $m$ th source segment and the $n$th receiver position, can be computed with precision. Similar equations can be obtained for combining other source segments and receiver positions. Consequently, for all source segments and receiver positions, a transfer matrix can be written as follows:

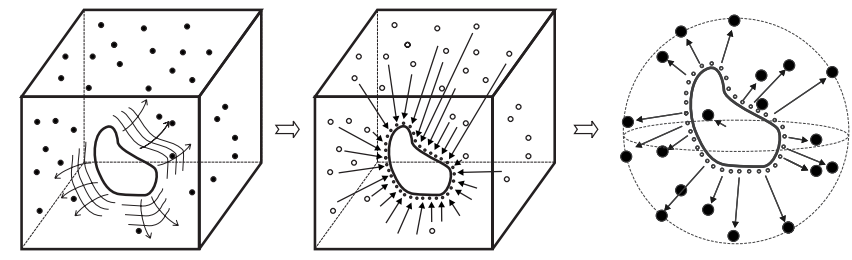

FIG. 3. An illustration for the sound power estimation. First, transfer functions are calculated and then backward reconstruction of the source pressure is carried out. With the estimated source pressure, the sound power of the source is calculated according to ISO 3745.

$$
\begin{gathered}
\left\{\begin{array}{c}
p_{1} \\
p_{2} \\
\vdots \\
p_{N}
\end{array}\right\}=\left\{\begin{array}{cccc}
H_{11} & H_{12} & \cdots & H_{1 M} \\
H_{21} & H_{22} & \cdots & H_{2 M} \\
\vdots & \vdots & \vdots & \vdots \\
H_{N 1} & H_{N 2} & \cdots & H_{N M}
\end{array}\right\}\left\{\begin{array}{c}
p_{s, 1} \\
p_{s, 2} \\
p_{s, 3} \\
\vdots \\
p_{s, M}
\end{array}\right\} \\
\text { or } \quad \mathbf{P}_{f, N \times 1}=\mathbf{H}_{N \times M} \mathbf{P}_{s, M \times 1} .
\end{gathered}
$$

From the measured field pressures of $\mathbf{P}_{f}$, the source pressures $\mathbf{P}_{s}$ can be inversely recovered from Eq. (3) as

$$
\mathbf{P}_{f, N \times 1}=\mathbf{H}_{N \times M} \mathbf{P}_{s, M \times 1} \Rightarrow \mathbf{P}_{s, M \times 1}=\mathbf{H}_{N \times M}^{-1} \mathbf{P}_{f, N \times 1} .
$$

Figure 3 briefly depicts the entire process of the study. First of all, an extended noise source with a priori known surface pressure distribution emits sound in a room. A transfer function matrix, $\boldsymbol{H}$, from source segments to receiver locations was calculated by the PBTM. As a result, noiseless field pressures, $\mathbf{P}_{f}$, are obtained by multiplying the transfer matrix with the known surface pressures according to Eq. (3). Measurement noise can be artificially added to the calculated $\mathbf{P}_{f}$. In a practical situation, field pressures are generally measured by a well-calibrated array technique. The second figure shows that surface pressures are reconstructed by the inversion of the calculated transfer matrix according to Eq. (4). This process is named reconstruction of source data. The reconstruction error must vanish in a noise-free condition, but it increases if the measurement noise is involved in. During this process, a regularization can be employed. For quantifying a reconstruction error, Monte Carlo simulations were conducted for pre-defined sets of field points. Once the surface pressures are reconstructed, the source data can be used to calculate the sound pressures at any receiver/field points. This process is called regeneration of sound field. The third figure shows a calculation of field pressures from the reconstructed source data as if it is situated in an anechoic chamber. The acoustic power of the source can be estimated by using the calculated field pressures.

\section{TEST EXAMPLE WITH OMNI-DIRECTIONAL SOURCES}

For the simplest case, two omni-directional sources were chosen. The first one is an icosahedron source in Fig. 4(a), which consists of 20 equilateral triangles. The other is a cubic source having 24 isosceles triangles in Fig. 4(b). Figure 4(c) shows a rectangular room model and 96 evenly spaced 


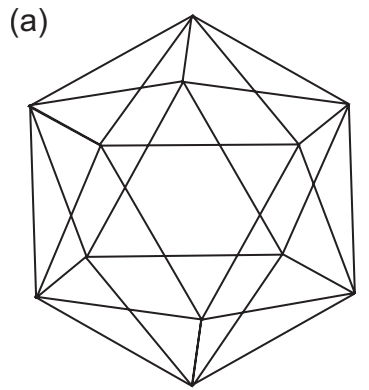

(b)

(c)

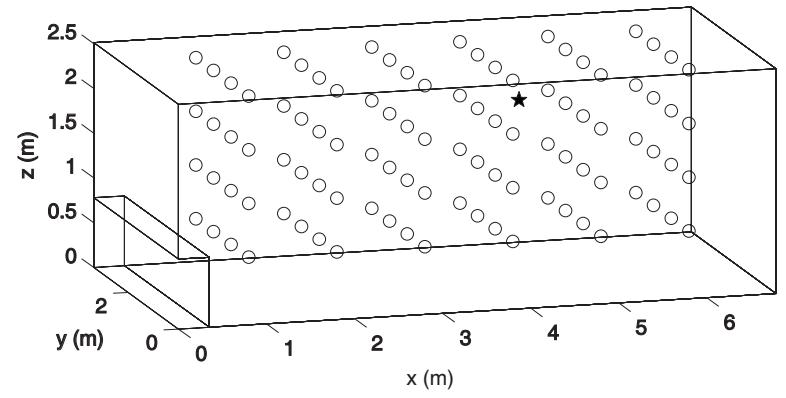

FIG. 4. Room and source model. (a) Icosahedron source, (b) cubic source, and (c) parallelepiped room. Locations of 96 candidates field points are shown as $\bigcirc$, whereas the symbol $\star$ denotes the source location.

field points in the target space. The size of the room is 6.8 $\times 3.4 \times 2.5 \mathrm{~m}^{3}$. The omni-directional source is located at $4.3,1.5$, and $2 \mathrm{~m}$.

Wall boundary conditions were given by absorption coefficients shown in Table I, and the angle-dependent reflection coefficients ${ }^{9,30}$ were calculated considering the size of the walls. The maximum number of reflection was limited to 50. Based on the measured reverberation time of $1.2 \mathrm{~s}$ at medium frequencies, the Schroeder cutoff frequency, ${ }^{10} f_{c}$, was about $300 \mathrm{~Hz}$. The common definition of medium frequency is from $f_{c}$ to $4 f_{c}{ }^{31}$ This study also focuses only on the medium frequencies due to the basic premise of the PBTM, although the method can be extendedly applicable to the outside of the medium frequency range with small inevitable errors. The selected medium frequency for demonstration was $900 \mathrm{~Hz}$, which corresponds to about $3 f_{c}$.

\section{A. Effect of field points and regularization with icosahedron source}

Twenty beams emanate from the icosahedron source, which means basically 1 beam per source segment was emitted and traced. In order to investigate the effect of field point locations, three sets of field points were chosen. As the first

TABLE I. Absorption coefficients of the room surfaces.

Surface (material)

Floor (stone)

Ceiling (gypsum)

Wall (concrete)

Window (glass)

Ventilation grating (partially open)

Ventilation cover (thin steel)
Absorption coefficient

0.02

0.04

0.02

0.04

0.60

0.05 (a)

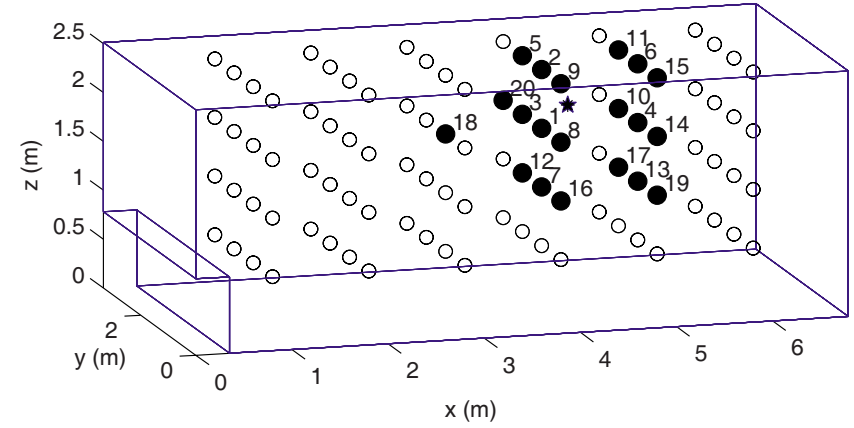

(b)

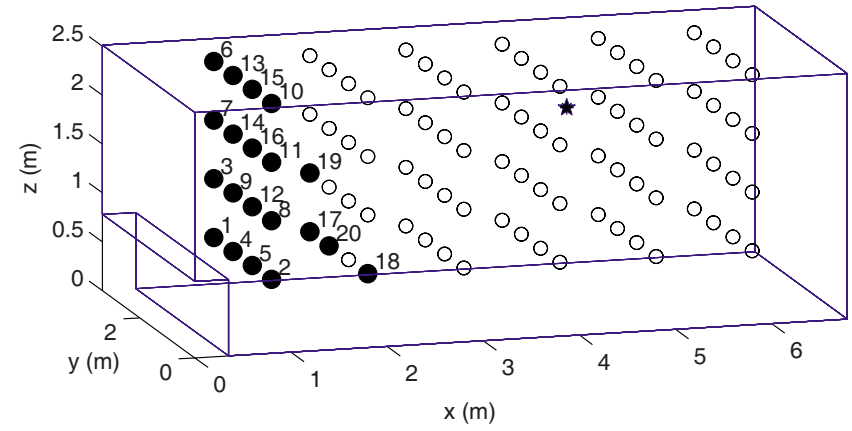

(c)

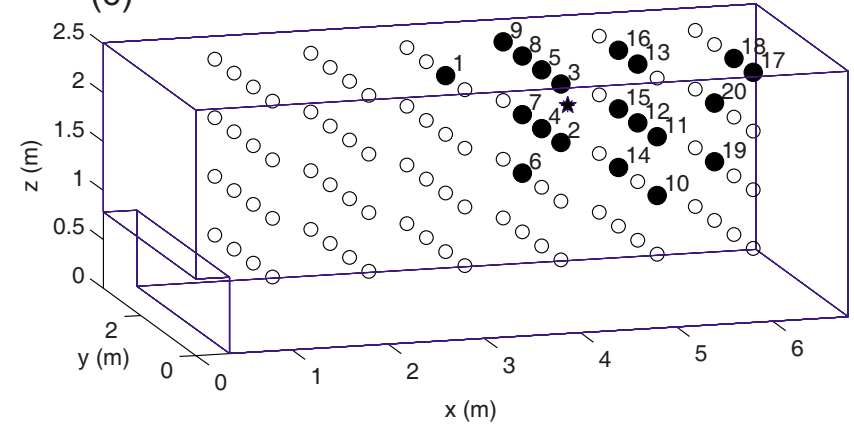

FIG. 5. (Color online) Locations of 20 field points. (a) Field point set 1, (b) field point set 2 , and (c) field point set 3 .

set, only 20 points among 96 candidate field points were chosen by excluding the farthest 76 points from the source, in Fig. 5(a). On the contrary, the 76 closest sensor positions to the source are eliminated in the second set as can be seen in Fig. 5(b). The third set consists of 20 sensor positions determined by the effective independent (EfI) technique in Fig. 5(c).

In the real situation, the backward reconstruction of the source field suffers from divergence phenomenon during the inverse of ill-conditioned matrix. The major cause of such an additional ill-conditioning, besides the effect of measurement noise, is originated from the redundancy of field points, which is reflected into the transfer matrix having dependency in between columns and rows. To assure the independence among field positions, a sensor positioning method, so called EfI method, can be employed in the initial setting of the field points. The method is based on the mathematical strategy of ranking the contribution of each candidate sensor location to the rank of the system matrix. This technique was already successfully applied to many inverse problems: for the wavebased identification of a large space structure ${ }^{32,33}$ and the 

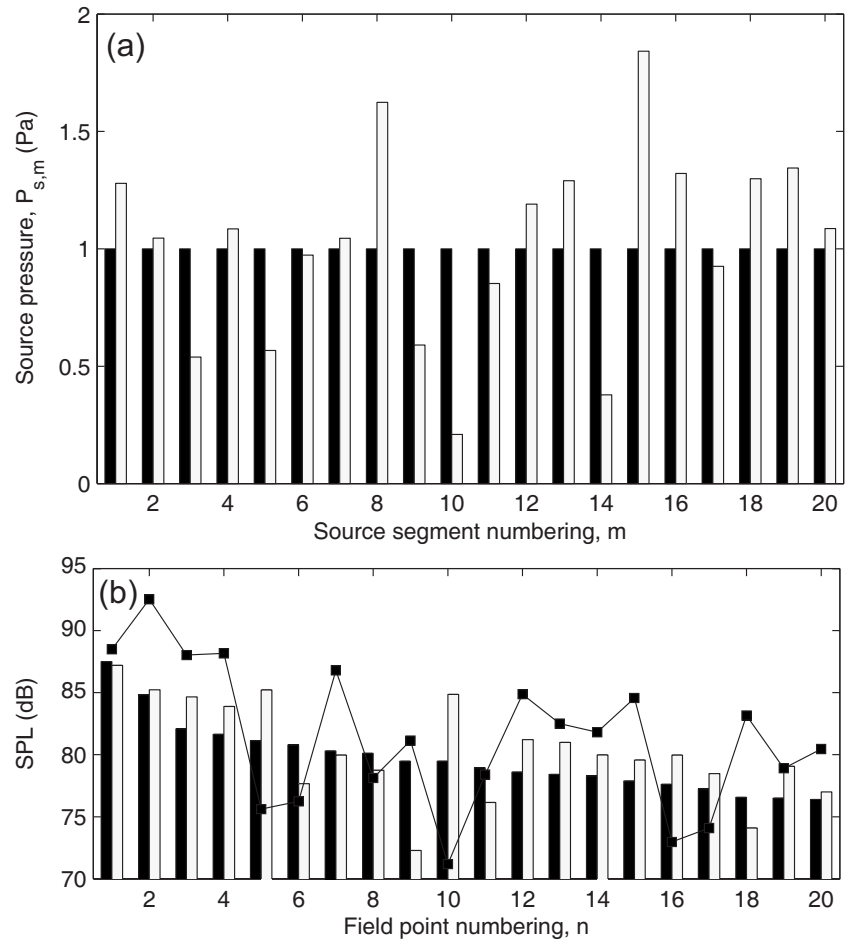

FIG. 6. Recovered source data and regenerated free-field data at set 1: , noise-free condition; $\square$, condition with $\mathrm{SNR}=20 \mathrm{~dB}$; and $\longrightarrow$ SPL in the presence of enclosure walls.

BEM-based NAH. ${ }^{34,35}$ First, an initial candidate set of sensor location is selected, which covers the whole acoustic domain in a very fine manner. Then, the transfer matrix $\boldsymbol{H}$ in Eq. (3) is analyzed by the singular decomposition technique, which will express the matrix $\boldsymbol{H}$ as a multiplication of two unitary singular vectors, $\boldsymbol{U}$ and $\boldsymbol{V}$, and a diagonal singular matrix, $\boldsymbol{\Lambda}$. An EfI value is defined by a diagonal value of the multiplication of the left singular vector, $\boldsymbol{U}$, and its Hermitian form. Taking a point or several points as a group, one should calculate the EfI value of that corresponding point and the calculation of EfI value is carried out for all points. Because the EfI concept is to identify the contribution of each sensor position to the linear independence of the whole matrix, a small EfI value, in a relative sense, means that the corresponding sensor point depends a lot on the other points. Consequently, locations having smallest EfI value of all should be discarded from the initial population of the sensor positions. The same process continues with the remaining candidate set of sensor positions until a predetermined number of sensors is reached. In this way, the singularity factor of the transfer matrix can be reduced significantly before applying any regularization method. Hereafter, the three sets of field points are called "set 1," "set 2," and "set 3," respectively.

All the segmental source pressures were given by $1 \mathrm{~Pa}$. The measurement noise, from environment and measurement system itself, will be always included in practice. Because a small noise in the field data will be amplified during the inverse process, the effect of noise on the reconstructed result cannot be overlooked. Field pressures were contaminated by random noises having the mean signal-to-noise ratio (SNR) of $20 \mathrm{~dB}$, which follows a normal distribution. For set 1 , the reconstructed source pressure distribution on the surface is shown in Fig. 6(a). One can find that the recon-
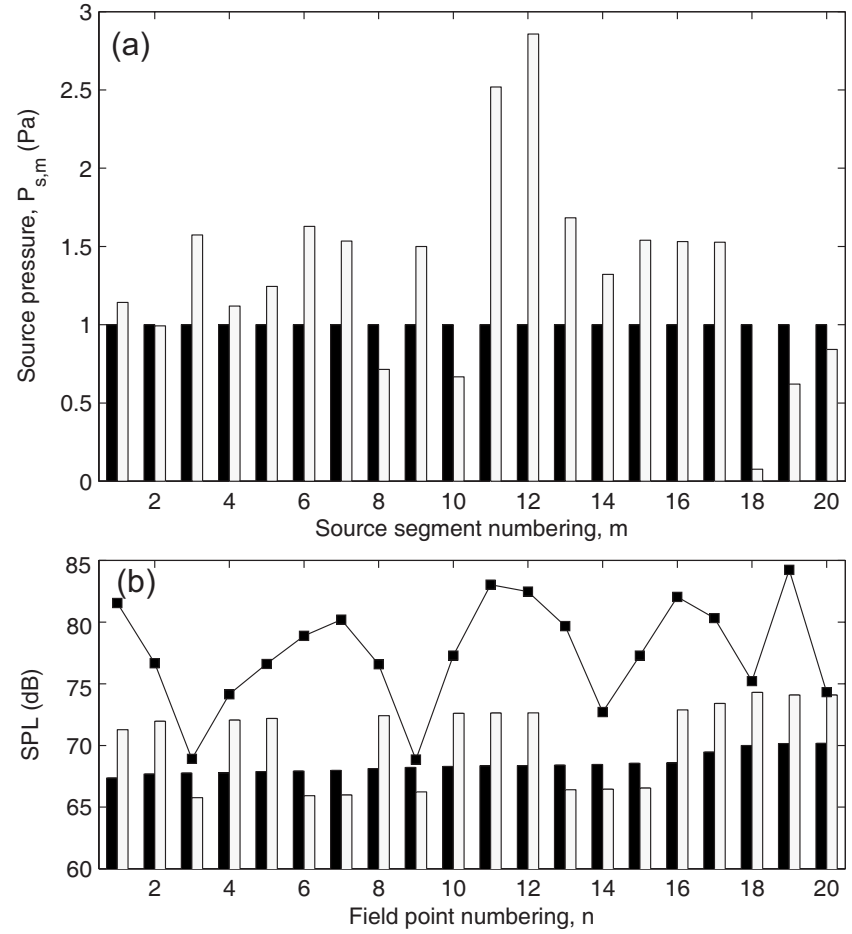

FIG. 7. Recovered source data and regenerated free-field data at set 2: $\square$, noise-free condition; $\square$, condition with $\mathrm{SNR}=20 \mathrm{~dB}$; and SPL in the presence of enclosure walls.

structed data recovered from the noise-contaminated field pressure data show a large difference from the original source pressure distribution: An estimated maximum error was $90 \%$ at 15 th source segment. To suppress the effect of measurement noise, thus enhancing the recovered source image, the regularization should be applied to the raw reconstructed data.

One can regenerate a sound field from the reconstructed source pressure. However, in regenerating pressures at the same field points in the same room from the "incorrect" recovered source data, a summation of the direct sound and successive reflections will end up with field pressures that are different from the original noise-contaminated field data. Therefore, in this back and forward calculation process, effect of room geometry is counted twice, viz., the reconstruction and regeneration. To avoid the double count of the wall reflection effect, the room surfaces are regarded invisible, in other words, as an anechoic condition in the regeneration. The calculated sound pressure levels at the initial field points are displayed in Fig. 6(b). In this figure, the field data without any measurement noise can be considered as a "true" data set. One can observe that the maximum difference in sound pressure levels is less than $5 \mathrm{~dB}$. The result shows that the overall distribution of the regenerated field data is similar to the initial data set. The line with square symbol denotes the calculated sound pressure level (SPL) when the wall reflections are taken into account.

The reconstructed source pressures from the field data at set 2 are shown in Fig. 7(a), which differ much from the original values. The regenerated sound pressure levels at field points set 2 are also different from those of noise-free condition, as can be seen in Fig. 7(b). SPL differences are 

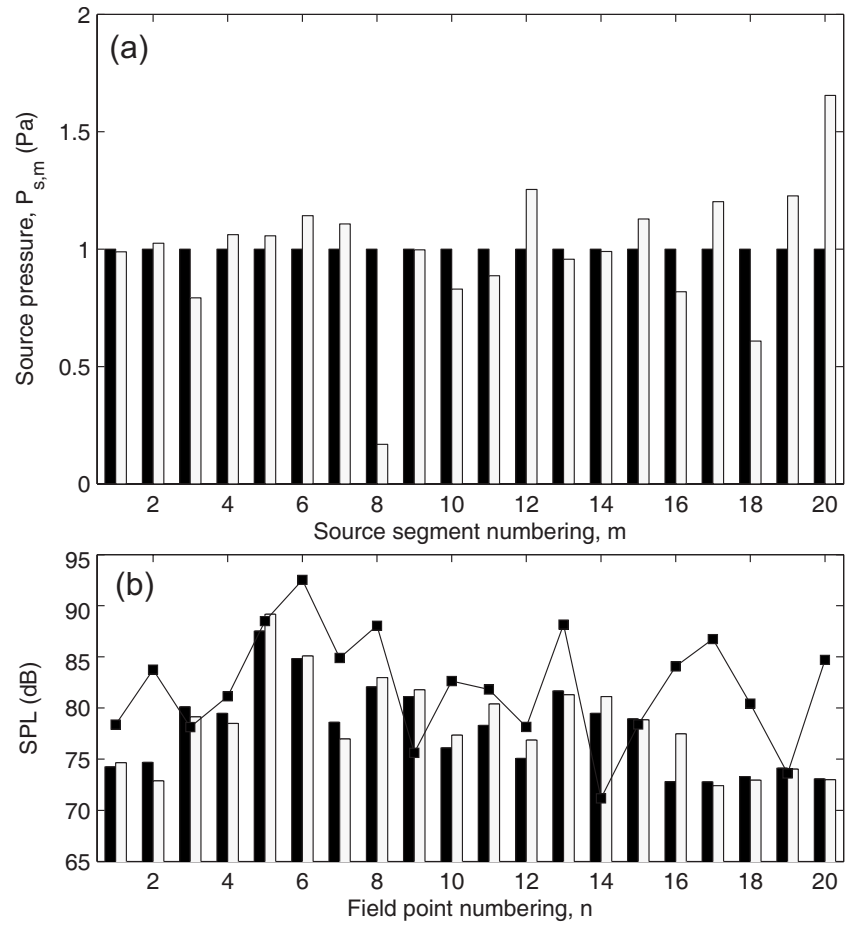

FIG. 8. Recovered source data and regenerated free-field data at set 3: noise-free condition; $\square$, condition with $\mathrm{SNR}=20 \mathrm{~dB}$; and $\longrightarrow$, SPL in the presence of enclosure walls.

magnified to $4 \mathrm{~dB}$ on average. This result emphasizes the importance of choosing proper locations of field sensor points. When the field points are far from the source, there is a tendency that the rank-deficient transfer matrix degrades the accuracy of the inverse problem, notwithstanding the fact that the far points from the source cannot always be regarded as bad points for the reconstruction.

Using the field pressure data measured at set 3 , the reconstructed source pressure distribution and the regenerated sound pressure levels at the field positions are depicted in Fig. 8. Apparently, the errors are much smaller than the other two cases using different field data sets.

To compare the errors, the Monte Carlo simulation was conducted for three field point sets with 5000 trials. A percentage error is defined as the ratio of difference in the surface pressure to the original surface pressure as

$$
\text { error }=\frac{1}{M} \sum_{m=1}^{M}\left|\frac{p_{s, m_{\text {noise }}}-p_{s, m_{\text {original }}}}{p_{s, m_{\text {original }}}}\right| \times 100(\%) .
$$

In Fig. 9, error histograms clearly show the importance of choosing appropriate field point locations. The average error for field points in set 3 amounts to 54\%, while the average errors by set 1 and set 2 correspond to $58 \%$ and $92 \%$, respectively.

In addition to the proper sensor placement technique, a regularization method was finally applied to overcome the instability of the inverse problem. The instability of the inverse reconstruction usually occurs due to the presence of small measurement noise and high order wave components, which are significantly amplified during the inversion. Due to this reason, the transfer matrix should be modified by
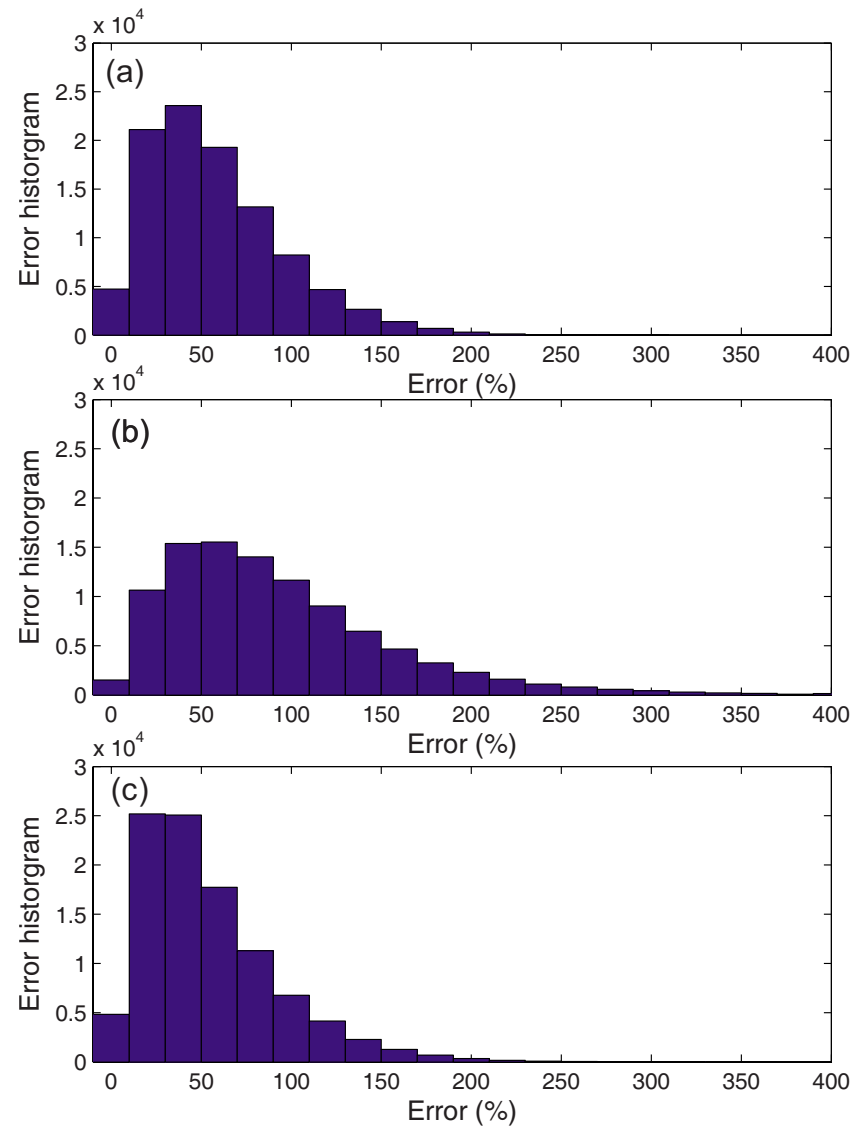

FIG. 9. (Color online) Error histograms of the reconstructed source pressures without regularization. (a) Field point set 1, (b) field point set 2, and (c) field point set 3 .

adopting an appropriate wave number filter. Among various regularization methods, the modified Tikhonov regularization method ${ }^{36,37}$ was applied to suppress the excessive effect of measurement noise. Regularization parameters were optimally determined by minimizing the generalized crossvalidation function. ${ }^{38}$

After applying the regularization method to the inverse process, a significant enhancement of the source image could be obtained as can be seen in Fig. 10. For poorly chosen field points, set 2, the average error was reduced to $38 \%$ as shown in Fig. 10(b). This is a dramatic improvement compared with the original average error of $92 \%$. The regularized results for field point set 1 and set 2 are similar in error distribution. The average error is of $37 \%$ when the field data set 1 is adopted, whereas the field data set 3 yield the lowest average error of $24 \%$.

\section{B. Cubic source}

So far, a spherical source has been adopted in the PBTM simulation. Because most machinery is shaped in the parallelepiped, the simulation using such a parallelepiped source would be meaningful for the practical applications of the present method. The cubic source in Fig. 4(b) was located at the same source location in the same enclosure in Fig. 4(c). In the simulation, the emitted number of beams was 24 , and the number of reflections was limited to 50. The absorption 

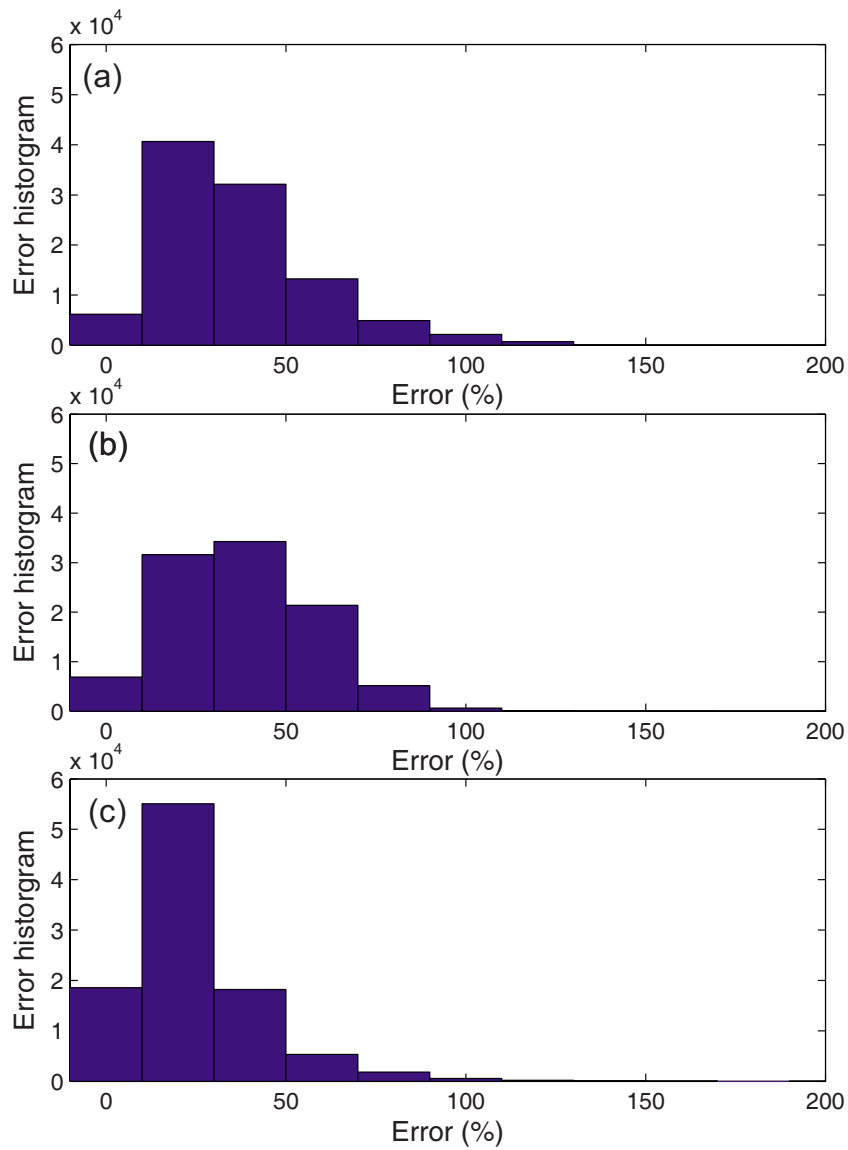

FIG. 10. (Color online) Error histograms of the reconstructed source pressures with the modified Tikhonov regularization method. (a) Field point set 1 , (b) field point set 2, and (c) field point set 3.

coefficients in Table I were used in the simulation. Figure 11 shows the regenerated sound pressure data, which yield a maximum error of $3 \mathrm{~dB}$.

\section{Estimation of sound power level}

Additional purpose of the present method was to estimate the sound power radiated from the source. When the field points were spherically distributed at a radius of $1 \mathrm{~m}$ from the source in an anechoic condition (see the rightmost part of Fig. 3), the sound power level could be estimated in accordance with ISO $3745 .{ }^{39}$ Sound pressures measured at predetermined 20 positions (Annex C of ISO 3745) were regenerated and the sound power level of the source was estimated as follows:

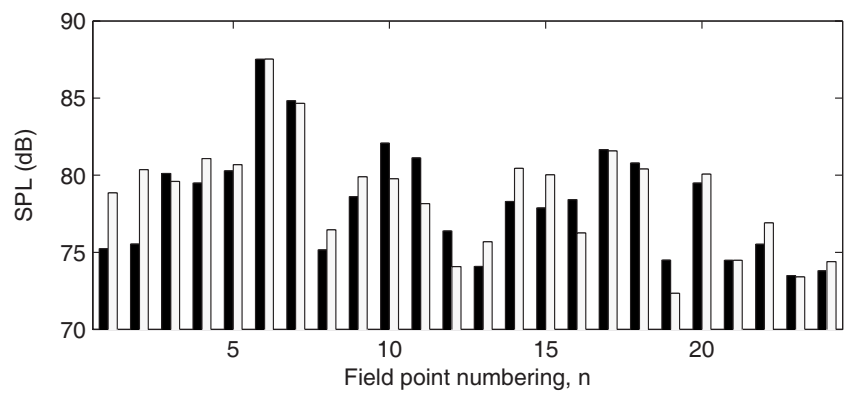

FIG. 11. Regenerated free-field data employing the cubic source: condition; $\square$, condition with $\mathrm{SNR}=20 \mathrm{~dB}$.

$$
L_{w}=\overline{L_{p}}+10 \log _{10}(4 \pi) .
$$

Here, $\overline{L_{p}}$ is the average sound pressure level over the 20 microphone locations, which is equivalent to the sound intensity level in an anechoic condition. In practice, however, one cannot precisely measure the free-field pressures without moving the source into an anechoic chamber.

It has been observed that the field pressures in the enclosure are generally higher than those in the free-field in Figs. 6(b), 7(b), and 8(b), mainly due to the interference of reflected waves from the walls. Because of these amplified field pressures in the enclosure, the sound power level of the source would be overestimated. For example, the estimated sound power of the source using the amplified field pressures was $94.3 \mathrm{~dB}$, whereas the actual sound power was $91.0 \mathrm{~dB}$. Using the regenerated free-field sound pressures by the PBTM, the average sound power level over 500 random trials was obtained as $91.4 \mathrm{~dB}$ with SNR of $20 \mathrm{~dB}$. This simulation shows a possibility to adopt the present method in estimating the sound power radiated from heavy and big noise sources located in factories or enclosures.

\section{CONCLUSIONS}

A method to inversely reconstruct sound source data within an enclosure was studied, which would be valid at medium frequencies. Using the phased beam tracing method, one could easily take the room effect into account in obtaining the transfer functions between the source segments and the field points. By solving the resultant inverse problem, it was shown that the source pressure could be recovered. EfI and a regularization technique were employed to overcome the inherent problem in the inversion process, thus enhancing the resultant source image. It is thought that the proposed technique for the source identification using the PBTM can be effectively applied to the estimation of the sound power level of noise source without moving a source to an ideal chamber. The present method will be experimentally validated and rather complicated room geometries and nonidealized pressure distributions of actual sources will be further investigated. Lastly, but not least, the controversial issue about adequacy of neglecting diffraction will be discussed in the future.

\section{ACKNOWLEDGMENT}

This work was partially supported by the BK21 Project and the NRL.

${ }^{1}$ J. S. Bendat and A. G. Piersol, Engineering Applications of Correlation and Spectral Analysis (Wiley, New York, 1980).

${ }^{2}$ F. J. Fahy, Sound Intensity (E \& FN Spon, London, 1976).

${ }^{3}$ J. D. Maynard, E. G. Williams, and Y. Lee, "Nearfield acoustic holography: I. Theory of generalized holography and the development of NAH," J. Acoust. Soc. Am. 78, 1395-1413 (1985).

${ }^{4}$ B.-K. Kim and J.-G. Ih, "On the reconstruction of vibro-acoustic field over the surface enclosing an interior space using the boundary element method," J. Acoust. Soc. Am. 100, 3003-3016 (1996).

${ }^{5}$ M. R. Bai, "Application of BEM (boundary element method)-based acoustic holography to radiation analysis of sound sources with arbitrarily shaped geometries," J. Acoust. Soc. Am. 92, 533-548 (1992).

${ }^{6}$ D. H. Johnson and D. E. Dudgeon, Array Signal Processing: Concepts and Techniques (Prentice-Hall, Englewood Cliffs, NJ, 1993). 
${ }^{7}$ Y. Kagawa, T. Tsuchiya, K. Fujioka, and M. Takeuchi, "Discrete Huygens' model approach to sound wave propagation-Reverberation in a room, sound source identification and tomography in time reversal," J. Sound Vib. 225, 61-78 (1999).

${ }^{8} \mathrm{~A}$. Wareing and M. Hodgson, "Beam-tracing model for predicting sound field in rooms with multilayer bounding surfaces," J. Acoust. Soc. Am. 118, 2321-2331 (2005).

${ }^{9}$ C.-H. Jeong, J.-G. Ih, and J. H. Rindel, "An approximate treatment of reflection coefficient in the phased beam tracing method for the simulation of enclosed sound fields at medium frequencies," Appl. Acoust. 69, 601613 (2008).

${ }^{10}$ M. R. Schroeder and H. Kuttruff, "On frequency response curves in rooms: Comparison of experimental, theoretical, and Monte Carlo results for the average frequency spacing between maxima," J. Acoust. Soc. Am. 34, 76-80 (1962).

${ }^{11}$ J. B. Allen and D. A. Berkley, "Image method for efficiently simulating small-room acoustics," J. Acoust. Soc. Am. 65, 943-950 (1979).

${ }^{12}$ H. Lee and B.-H. Lee, "An efficient algorithm for the image model technique," Appl. Acoust. 24, 87-115 (1988).

${ }^{13}$ A. Krokstad, S. Stroem, and S. Soersdal, "Calculating the acoustical room response by the use of a ray tracing technique," J. Sound Vib. 8, 118-125 (1968).

${ }^{14}$ A. Kulowski, "Algorithmic representation of the ray tracing technique," Appl. Acoust. 18, 449-469 (1985).

${ }^{15}$ A. Kulowski, "Error investigation for the ray tracing technique," Appl. Acoust. 15, 263-274 (1982).

${ }^{16} \mathrm{~T}$. Lewers, "A combined beam tracing and radiant exchange computermodel of room acoustics," Appl. Acoust. 38, 161-178 (1993).

${ }^{17}$ T. Funkhouser, N. Tsingos, I. Carlbom, G. Elko, M. Sondhi, J. E. West, G. Pingali, P. Min, and A. Ngan, "A beam tracing method for interactive architectural acoustics," J. Acoust. Soc. Am. 115, 739-756 (2004).

${ }^{18}$ I. A. Drumm and Y. W. Lam, "The adaptive beam-tracing algorithm," J. Acoust. Soc. Am. 107, 1405-1412 (2000).

${ }^{19} \mathrm{D}$. van Maercke and J. Martin, "The prediction of echograms and impulse responses within the Epidaure software," Appl. Acoust. 38, 93-114 (1993).

${ }^{20}$ E. De Geest and H. Patzold, "Comparison between room transmission functions calculated with a boundary element method and a ray tracing method including phase," Proceedings of Inter-Noise (1996), Vol. 96, pp. $3177-3180$.

${ }^{21}$ J. S. Suh and P. A. Nelson, "Measurement of transient response of rooms and comparison with geometrical acoustic models," J. Acoust. Soc. Am. 105, 2304-2317 (1999).

${ }^{22}$ N. Tsingos, I. Carlbom, G. Elko, R. Kubli, and T. Funkhouser, "Validating acoustical simulations in the Bell Labs box," IEEE Comput. Graphics Appl. 22, 28-37 (2002).

${ }^{23}$ R. G. Kouyoumjian and P. H. Pathak, "A uniform geometrical theory of diffraction for an edge in a perfectly conducting surface," Proc. IEEE 62, 1448-1461 (1974).

${ }^{24}$ A. D. Pierce, Acoustics. An Introduction to Its Physical Principles and Applications (American Institute of Physics, New York, 1991).

${ }^{25}$ N. Tsingos, T. Funkhouser, A. Ngan, and I. Carlbom, "Modeling acoustics in virtual environments using the uniform theory of diffraction," Proceedings of ACM SIGGRAPH (2001), CD-ROM.

${ }^{26}$ R. R. Torres, U. P. Svensson, and M. Kleiner, "Computation of edge diffraction for more accurate room acoustics auralization," J. Acoust. Soc. Am. 109, 600-610 (2001).

${ }^{27} \mathrm{C}$.-H. Jeong and J.-G. Ih, "Introduction and applications of phased beam tracing method: Can we interpret low frequency response by the particle property?," Proceeding of the International Congress on Acoustics (2007), Paper No. RBA-05-016.

${ }^{28}$ U. P. Svensson and R. I. Fred, "An analytic secondary source model of edge diffraction impulse responses," J. Acoust. Soc. Am. 106, 2331-2344 (1999).

${ }^{29}$ V. Pulkki, U. P. Svensson, and T. Paatero, "Efficient representation of edge diffraction impulse responses," Proceedings of the International Congress on Acoustics (2007), Paper No. RBA-05-014.

${ }^{30}$ J. H. Rindel, "Modeling the angle-dependent pressure reflection factor," Appl. Acoust. 38, 223-234 (1993).

${ }^{31}$ F. A. Everest, Master Handbook of Acoustics (McGraw-Hill, New York, 2001).

${ }^{32}$ D. C. Kammer, "Sensor placement for on-orbit modal identification and correlation of large space structures," J. Guid. Control Dyn. 14, 251-259 (1991).

${ }^{33}$ D. C. Kammer, “"'Effects of noise on sensor placement for on-orbit modal identification of large space structures," ASME J. Dyn. Syst., Meas., Control 114, 436-443 (1992).

${ }^{34}$ B.-K. Kim and J.-G. Ih, "Design of an optimal wave-vector filter for enhancing the resolution of reconstructed source field by near-field acoustical holography," J. Acoust. Soc. Am. 107, 3289-3297 (2000).

${ }^{35}$ I.-Y. Jeon and J.-G. Ih, "On the holographic reconstruction of vibroacoustic fields using equivalent sources and inverse boundary element method," J. Acoust. Soc. Am. 118, 3473-3482 (2005).

${ }^{36}$ E. G. Williams, "Regularization methods for near-field acoustical holography," J. Acoust. Soc. Am. 110, 1976-1988 (2001).

${ }^{37}$ G. H. Golub and C. F. V. Loan, Matrix Computations (Johns Hopkins University Press, Baltimore, MD, 1996).

${ }^{38}$ P. C. Hansen, Rank-Deficient and Discrete Ill-Posed Problems (SIAM, Philadelphia, PA, 1998).

${ }^{39}$ ISO 3745:2003, "Acoustics-Determination of sound power levels of noise sources using sound pressure-precision methods for anechoic and hemi-anechoic rooms" (International Organization for Standardization, Geneva, 2003). 